OPEN ACCESS

Edited by:

Massimo Gadina,

National Institute of Arthritis and Musculoskeletal and Skin Diseases (NIAMS), United States

Reviewed by:

Scott W. Canna,

University of Pittsburgh Medica

Center, United States Jiacai Cho,

National University Hospital, Singapore

*Correspondence:

Fabien Touzot

fabien.touzot@umontreal.ca

tThese authors have contributed equally to this work

Specialty section:

This article was submitted to Inflammation,

a section of the journal

Frontiers in Immunology

Received: 27 July 2018 Accepted: 03 September 2018 Published: 24 September 2018

Citation: Barsalou J, Blincoe A, Fernandez I, Dal-Soglio D, Marchitto L, Selleri S, Haddad $E$, Benyoucef $A$ and Touzot $F$ (2018) Rapamycin as an Adjunctive

Therapy for NLRC4 Associated

Macrophage Activation Syndrome.

Front. Immunol. 9:2162.

doi: 10.3389/fimmu.2018.02162

\section{Rapamycin as an Adjunctive Therapy for NLRC4 Associated Macrophage Activation Syndrome}

\author{
Julie Barsalou ${ }^{1 \dagger}$, Annaliesse Blincoe ${ }^{1 \dagger}$, Isabel Fernandez ${ }^{2,3,4}$, Dorothée Dal-Soglio ${ }^{3,5}$, \\ Lorie Marchitto $^{3,4}$, Silvia Selleri ${ }^{4}$, Elie Haddad ${ }^{1,3,4}$, Aissa Benyoucef ${ }^{4}$ and Fabien Touzot ${ }^{1,3,4 *}$ \\ ${ }^{1}$ Department of Immunology-Rheumatology, Department of Pediatric, CHU Sainte Justine, Montréal, QC, Canada, \\ 2 Immunology Laboratory, CHU Sainte Justine, Montréal, QC, Canada, ${ }^{3}$ Microbiology, Infectiology and Immunology \\ Department, Université de Montréal, Montréal, QC, Canada, ${ }^{4} \mathrm{CHU}$ Sainte Justine Research Center, Montréal, QC, Canada, \\ ${ }^{5}$ Pathology and Cellular Biology Department, CHU Sainte Justine, Université de Montréal, Montréal, QC, Canada
}

Gain of function (GOF) mutations affecting the inflammasome component NLRC4 are known to cause early-onset macrophage activation syndrome (MAS) and neonatal enterocolitis. Here we report a patient with a NLRC4 GOF mutation presenting with neonatal MAS efficiently treated with a combination of anakinra and rapamycin. Through in vitro studies, we show that rapamycin reduces both IL-1 $\beta$ and IL-18 secretion by the patient's phagocytic cells. The reduction of cytokine secretion is associated with a reduction of caspase- 1 activation regardless of the pathogen- or danger-associated molecular patterns triggering the activation of the inflammasome. This study suggests that patients with inherited auto-inflammatory disorders could benefit from an adjunctive therapy with rapamycin.

Keywords: macrophage activation syndrome, NLRC4, inflammasome, mTOR, rapamycin, IL-18, IL-1 $\beta$

\section{INTRODUCTION}

Our patient is a male infant, born at $35+4$ weeks of gestation to non-consanguineous parents. He was admitted to the intensive care unit at 12 days of age with profuse bloody diarrhea, weight loss, severe metabolic acidosis and acute renal failure. He developed a vasoplegic shock necessitating intubation, ventilation and inotropic support. The patient developed features of macrophage activation syndrome (MAS)/Hemophagocytic Lymphohistiocytosis (HLH) with: (i) prolonged fever $>38.5^{\circ} \mathrm{C}$, (ii) hepatosplenomegaly, (iii) anemia (hemoglobin $9.7 \mathrm{~g} / \mathrm{dL}$ ) and thrombocytopenia (platelets $34 \times 10^{9} / \mathrm{L}$ ), (iv) hypertriglyceridemia (triglycerides $4.8 \mathrm{mmol} / \mathrm{L}$ ), (v) hyperferritinemia $(14,7000 \mathrm{ng} / \mathrm{mL}$ ), and (vi) haemophagocytosis on bone marrow biopsy. He also presented with three remarkable features (i) a macular erythematous rash that slowly resolved and was replaced by a reticulo-livedoid rash, (ii) a marked hypereosinophilia (up to $2.4 \times 10^{9} / \mathrm{L}$ ) and (iii) no significant elevation of HLA-DR/CD8 ${ }^{+}$T-cells on lymphocyte immunophenotyping. Stool microscopy showed presence of necrotic intestinal mucosa with absence of inflammatory cells (Figure 1E). Rectal biopsy demonstrated the presence of eosinophils with no significant inflammatory cell infiltrate nor architectural changes (Figure 1D). Immunohistochemistry staining performed with anti-IL18 antibody (HPA003980, Sigma) revealed moderate IL-18 staining of surface epithelium and crypts (Figure 1F). Immune work-up demonstrated global T cell lymphopenia with a balanced subpopulation. Expression of perforin and CD107a expression on NK cells was normal. Circulating FOXP $3{ }^{+} \mathrm{CD} 25^{+} \mathrm{CD} 127^{\text {low }} \mathrm{CD} 4^{+}$T-cells were within normal range. A dihydrorhodamine reduction 
assay was normal. Subsequent genetic analysis identified the presence of a de novo heterozygous mutation in the nucleotide binding domain (NBD) of NLRC4 (c.1021G>C, p.Val341Leu). This mutation is an alternate substitution of a previously reported mutation (p.Val341Ala) in a boy who presented with NLRC4 associated MAS (1).

We demonstrated the spontaneous activation of NLRC4 in vitro as evidenced by the high level of IL-1 $\beta$ (317 \pm 168 pg/mL vs. $5 \pm 2 \mathrm{pg} / \mathrm{mL}, p=0.0007)$ and IL-18 (205 \pm 175 $\mathrm{pg} / \mathrm{mL}$ vs. $0 \mathrm{pg} / \mathrm{mL}, p<0.0001)$ secretion by unstimulated monocyte-derived macrophages (MDM) as compared to healthy controls (HC) (Figure 2A). Of note, we also observed higher secretion of IL-1 $\beta$ and IL-18 by the patient's MDM after sequential stimulation by lipopolysaccharide (LPS) and different NLRP3 [adenosine triphosphate (ATP), monosodium urate crystal (MSU) or nigericin] and NLRC4 (lipotransfected flagellin) activators as compared to MDM from HC (Figure 2A).

The patient was initially treated with IV methylprednisone ( $2 \mathrm{mg} / \mathrm{kg} /$ day) and anakinra (up to $15 \mathrm{mg} / \mathrm{kg} /$ day). We decided to introduce rapamycin as a steroid-sparing agent reasoning that this drug could (i) potentiate the action of anakinra through autophagy induction and (ii) counteract the effect of IL-18 on T-cells through mTOR inhibition (2-5). The targeted trough concentration of rapamycin was $10-15 \mathrm{ng} / \mathrm{mL}$. The patient showed clinical response to the therapy within a week, allowing weaning of steroids, establishment of enteral feeds and weight gain. The C-reactive protein and ferritin dropped to $15-30 \mathrm{mg} / \mathrm{L}$ and $250-300 \mathrm{ng} / \mathrm{mL}$ respectively (Figure $1 \mathrm{C}$ ). Relative proportions of $\mathrm{CD} 14^{+} \mathrm{CD} 16^{-}$"classical," $\mathrm{CD} 14^{+} \mathrm{CD} 16^{+}$ "intermediate" and $\mathrm{CD} 14^{\text {low }} \mathrm{CD} 16^{+}$"non-classical” monocytes also normalized under therapy (Figure 1B) (6). Intriguingly, we documented a marked decrease of IL-18 (from 82844 to $9776 \mathrm{ng} / \mathrm{mL}$, Figure 1C) in the patient's plasma, whereas IL$1 \beta$ levels remained undetectable throughout the follow-up. This feature suggested a specific effect of rapamycin on IL-18 secretion. Previous reports of patients with NLRC4 GOF have indeed shown chronic elevation of serum IL-18 despite aggressive anti-inflammatory and IL-1 receptor blockade therapy (7). Of note, a rectal biopsy performed 6 months after initiation of rapamycin therapy showed persistent IL-18 staining (data not shown).

\section{BACKGROUND}

Inflammasomes are innate immune complexes that respond to pathogen- and danger-associated molecular patterns (PAMP and DAMP) through caspase-1 activation and interleukin (IL)$1 \beta$ and IL-18 secretion (8). Recently, gain of function (GOF) mutations affecting the NLRC4 inflammasome component have been reported to cause early-onset recurrent fever and MAS $(1,9)$.

Most reported mutations are located in the NBD of the protein (Figure 1A). The mutations are thought to disrupt the interaction between the leucine-rich repeat (LRR) domain and the NBD, facilitating NLRC4 conformational changes and its steady activation (10).
IL-1 $\beta$ blockade is effective in most inherited disorders associated with inflammasome dysregulation. However, the precise role of IL-1 $\beta$ in the development of MAS remains controversial (11). Interestingly, NLRC4 GOF has emphasized the important role of IL-18 in the development of MAS (12). In this disease, IL-18 secretion depends not only on phagocytic cells but also on epithelial cells $(12,13)$. Management of MAS associated with NLRC4 GOF mutations remains challenging. Minimal or absent response to anakinra has been described in patients with early onset disease $(7,11,14)$ Recombinant IL18BP (tadekinig- $\alpha$ ) has shown promising results in a patient with NLRC4 GOF mutation and is under evaluation in a phase 3 study (NCT03113760) (7). Allogeneic hematopoietic stem cell transplantation (HSCT) is the treatment of choice for primary HLH, however to date there have been no published report published of such treatment in patients with NLRC4 GOF mutations (15). HSCT may indeed cure the hyperinflammation driven by the immune system, but it is unlikely to have significant impact on the intestinal disease nor the IL-18 hypersecretion by the intestinal epithelial cells $(12,13)$.

\section{DISCUSSION}

The results presented here constitute the first in vivo and in vitro evidence of the anti-inflammatory effect of mTOR inhibitors in inherited inflammasome disorders. Patients with NLRC4 GOF mutations remain difficult to manage due to the severity of disease and the partial response to therapy $(1,9)$. We therefore decided to introduce rapamycin as adjunctive therapy reasoning that this drug could (i) potentiate the action of anakinra through autophagy induction and (ii) counteract the effect of IL-18 on T-cells through mTOR inhibition (2-4). The clinical and biochemical improvement of our patient mirrored the effects of rapamycin seen on MDM from the patient in vitro. While rapamycin had no remarkable effect on the spontaneous secretion of IL-1 $\beta$ by the patient's MDM during the first 6 days of differentiation culture prior to further stimulation, it significantly reduced their IL-18 secretion (Figure 2A). This could be explained by (i) different mechanisms through which rapamycin counteracts IL-1 $\beta$ and IL-18 cleavage and secretion or (ii) differences in epigenetic and transcriptional regulation of the $I L 1 B$ and IL18 loci. Of note, rapamycin also significantly reduced the secretion of both IL- $1 \beta$ and IL-18 by the patient's MDM after sequential activation with LPS plus activators of the NLRP3 and NLRC4 inflammasomes (Figures 2A,B). This reduction in cytokine secretion was associated with reduction of caspase-1 activation-as evidenced by FLICA-Caspase 1 assay-regardless of the PAMP or DAMP involved in inflammasome activation (Figures 2B,C). Interestingly, the same effect of rapamycin was observed in MDM derived from the patient with the p.His392del mutation and in MDM from healthy donors (Figure 2), showing that the effect of rapamycin was not restricted to the reported patient. Unfortunately, we could not demonstrate a reduction of IL-18 secretion by rapamycin in rectal epithelial cells because 

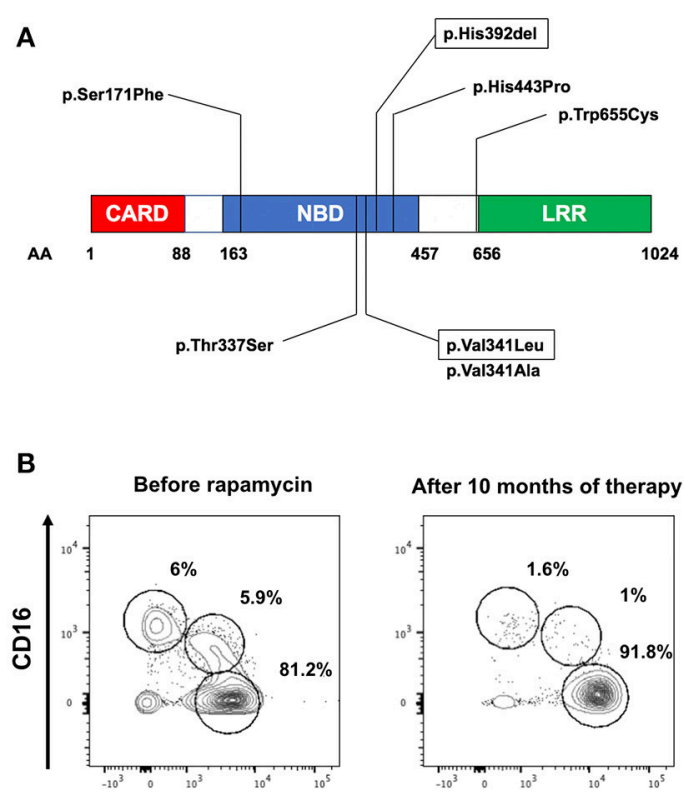

After 10 months of therapy

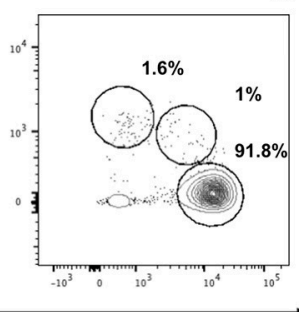

CD14

D

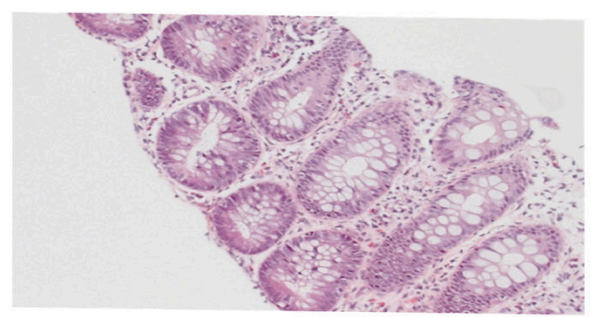

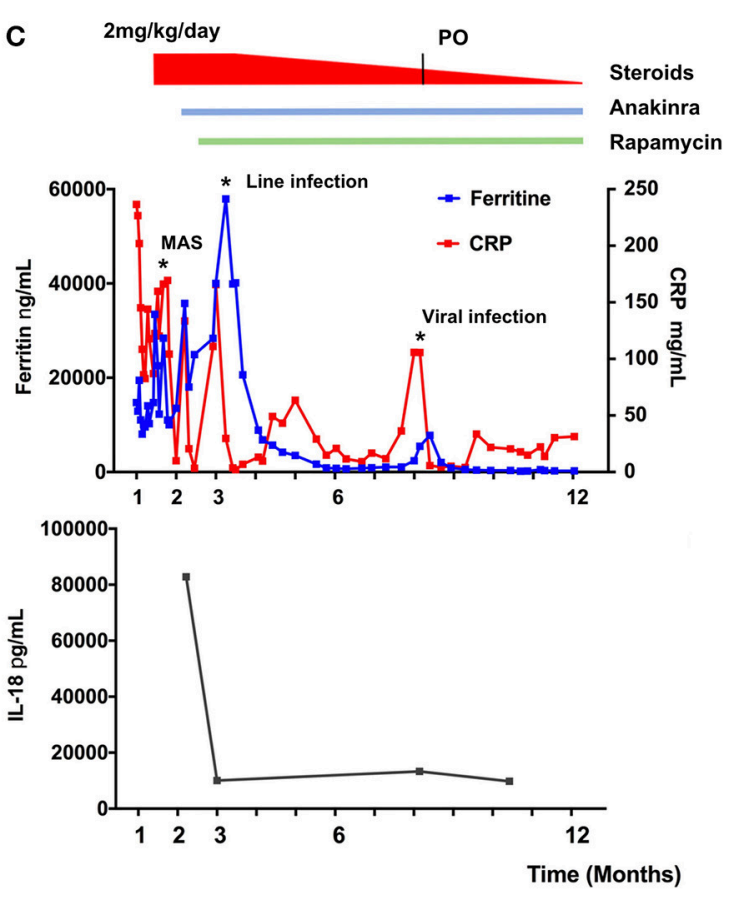

E

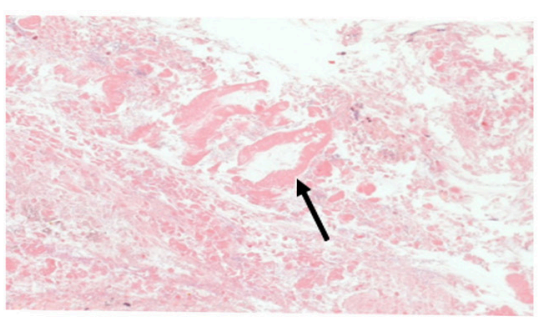

F

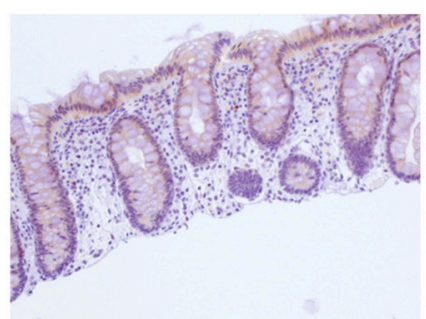

Healthy rectum

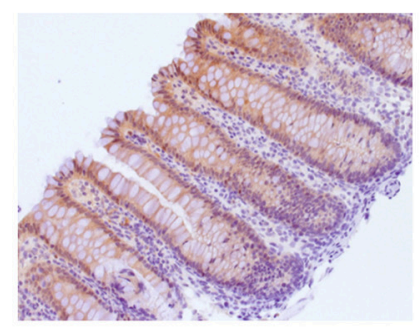

Patient NLRC4 p.V341L rectum

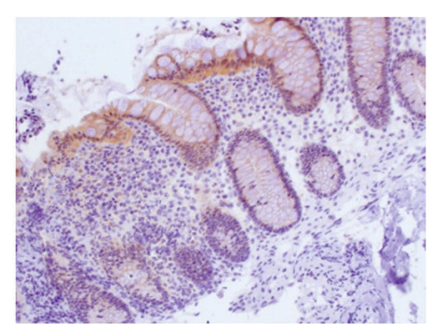

Ulcerative colitis colon

FIGURE 1 | Effect of rapamycin treatment in vivo on a patient with pVal341Leu NLRC4 mutation. (A) Reported NLRC4 mutations associated with macrophage activation syndrome are located in the Nucleotide Binding Domain. Mutation p.Val341Leu and p.His392del are framed. (B) Evaluation of circulating monocytes subsets in the patient peripheral blood before and after initiation of therapy by flow cytometry. (C) Ferritin, C-reactive protein (CRP) and IL-18 levels in sera of the patient. MAS (macrophage activation syndrome). Line infection was caused by staphylococcus epidermidis. Viral infection was rhinopharyngitis without microbial documentation. (D) Hematoxylin and eosin staining of rectal biopsy from the patient. (E) Stool microscopy. Arrow point to necrotic intestinal mucosa. (F) IL-18 staining of rectal biopsy from healthy control (left panel), patient with NLRC4 p.V341L mutation (middle panel) and colon biopsy of a patient with ulcerative colitis (left panel).

of the non-quantitative nature of the immunohistochemistry staining.

Altogether, these clinical and in vivo and in vitro biological data demonstrate for the first time that rapamycin is an interesting adjunctive therapy for patients with NLRC4 GOF mutations though reduction of both IL-1 $\beta$ and IL-18 secretion by phagocytic cells. This reduction of cytokine secretion is associated with a reduction of caspase- 1 activation but the precise mechanism through which rapamycin inhibits inflammasome activation remains to be determined $(3,13)$. This effect could 


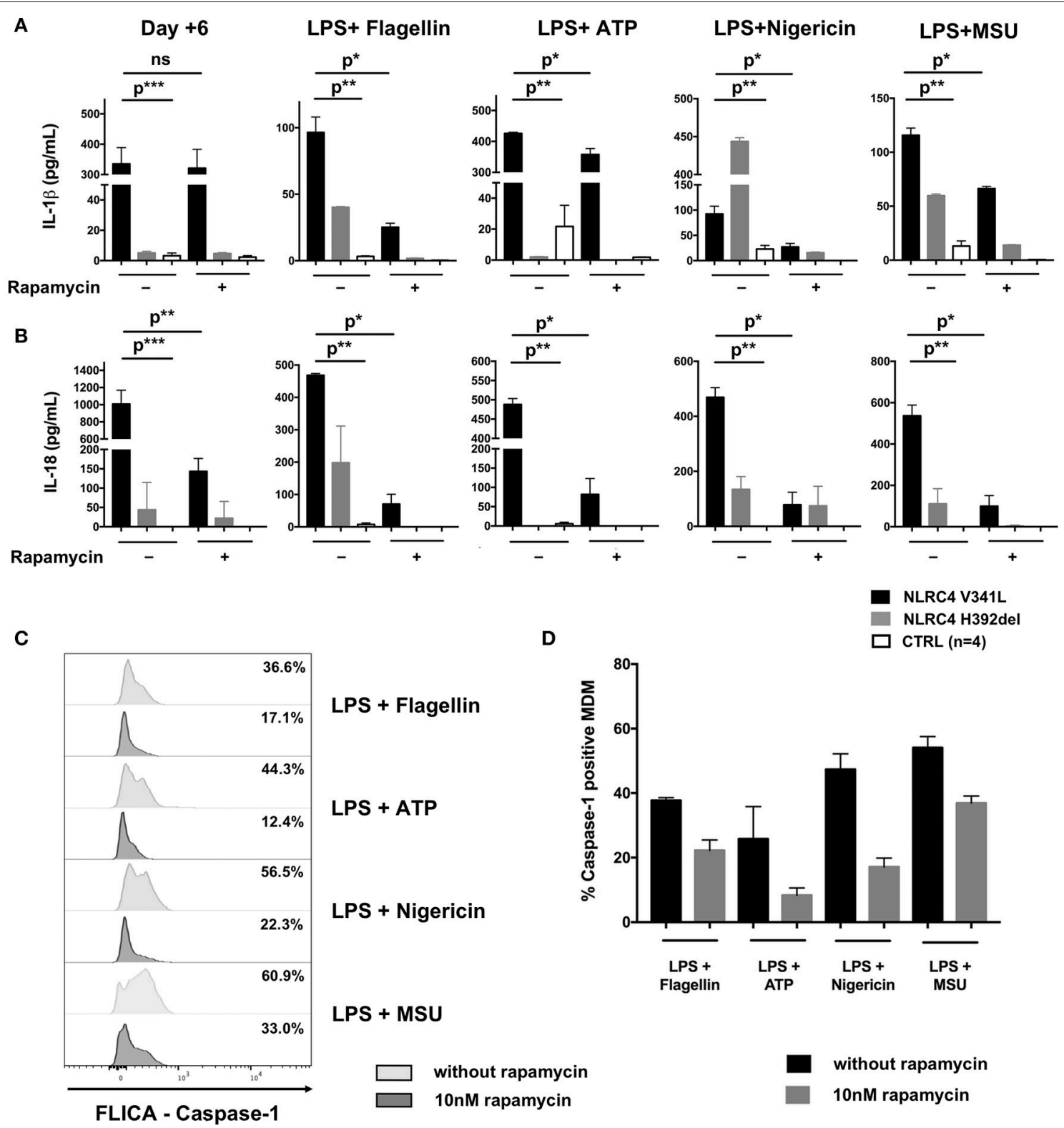

FIGURE 2 | Effect of rapamycin in vitro in macrophage-derived monocytes from a patient with NLRC4 GOF mutations. CD14+ monocytes from patients with p.Val341Leu mutation (3 samples in duplicate) and p.His392del mutation (2 samples in duplicates) in NLRC4, and healthy controls ( $n=4,4$ samples in duplicates) were differentiated into macrophages with or without rapamycin. Supernatant were collected at day +6 after differentiation and after stimulation of differentiated macrophage with NLRP3 (ATP, nigericin, MSU) or NLRC4 (lipotransfected flagellin) activators. IL-1 $\beta$ (A) and IL-18 (B) were analyzed by ELISA. (C) Active caspase-1 in MDM from the NLRC4 patient with p.Val341Leu mutation treated or not with $10 \mathrm{nM}$ rapamycin quantified by caspase-1 FLICA after stimulation with NLRP3 (ATP, nigericin, MSU) or NLRC4 (lipotransfected flagellin) activators. (D) Active caspase-1 in MDM from patients with p.Val341Leu mutation (2 samples) and p.His392del mutation in NLRC4 (2 samples in duplicates), treated or not with $10 \mathrm{nM}$ rapamycin quantified by caspase-1 FLICA after stimulation with NLRP3 (ATP, nigericin, MSU) or NLRC4 (lipotransfected flagellin) activators. $p^{\star}<0.05 ; p^{\star \star}<0.01, p^{\star \star \star}<0.001$; ns not significant (Man-Whitney T-test or Wilcoxon paired $T$-test).

involve diverse counter-regulatory pathways in phagocytes and perhaps also in intestinal epithelial cells $(3,13)$.

\section{CONCLUDING REMARKS}

We report herein a patient with a NLRC4 GOF mutation presenting with neonatal MAS efficiently treated with a combination of anakinra and rapamycin. The mTOR inhibitor reduced secretion of both IL- $1 \beta$ and IL-18 by the patient's phagocytic cells, regardless of the PAMP or DAMP triggering the inflammation, through reduction of caspase-1 activation. Our report paves the way for the use of mTOR inhibitors in the management of patients presenting with inherited inflammasome disorders. 


\section{Methods}

\section{Macrophage Differentiation}

Monocytes were isolated from PBMCs by negative selection using the EasySep ${ }^{\mathrm{TM}}$ Human Monocyte Isolation Kit (19058, Stem Cell Technology) according to the manufacturer's instructions. Monocytes were differentiated in Stemspan Medium with $100 \mathrm{ng} / \mathrm{mL}$ of recombinant human Granulocyte-Macrophage Colony-Stimulating Factor (Peprotech) for 6 days with or without $10 \mathrm{nM}$ rapamycin (R8781, Sigma) (added at day 0 of culture and at day 3 without changing the medium).

\section{Macrophage Secretion Assay}

After 6 days of differentiation, MDM were stimulated for $3 \mathrm{~h}$ by lipopolysaccharide (LPS) followed by stimulation with different activators of NLRP3 or NLRC4 inflammasome $(2.5 \mathrm{mM}$ ATP for $45 \mathrm{~min} ; 250 \mu \mathrm{g} / \mathrm{ml} \mathrm{MSU}, 2.5 \mu \mathrm{M}$ nigericin, and $1 \mu \mathrm{g} / \mathrm{ml}$ of lipotransfected flagellin, all for $4 \mathrm{~h}$ ) in presence or absence of $10 \mathrm{nM}$ rapamycin. The caspase- 1 activity was assessed by flow cytometry using caspase-1 FLICA (ICT098, Bio-Rad) according to the manufacturer's instructions.

IL- $1 \beta$ and IL-18 were measured in plasma from patient and culture medium by ELISA (88-7261-88 and BMS267-2 from eBioscience). IL-18 analysis in plasma of patients required a $25-$ fold dilution to allow measurement in the linear part of the standard curve.

\section{Immunohistochemistry}

IL-18 immunohistochemistry staining was performed with antiIL18 antibody (HPA003980, Sigma) according to standard procedures.

\section{Monocytes Subset Analysis}

Monocytes subset analysis was performed on total fresh blood using the following antibodies: anti CD16 PeCy7, anti-CD14 VioBlue, anti-HLA-DR FITC from BD biosciences.

\section{REFERENCES}

1. Romberg N, Al Moussawi K, Nelson-Williams C, Stiegler AL, Loring E, Choi M, et al. Mutation of NLRC4 causes a syndrome of enterocolitis and autoinflammation. Nat Genet. (2014) 46:1135-9. doi: 10.1038/ng.3066

2. Gabrion A, Hmitou I, Moshous D, Neven B, Lefèvre-Utile A, Diana J-S, et al. Mammalian target of rapamycin inhibition counterbalances the inflammatory status of immune cells in patients with chronic granulomatous disease. $J$ Allergy Clin Immunol. (2017) 139:1641-9.e6. doi: 10.1016/j.jaci.2016.08.033

3. Ko JH, Yoon S-O, Lee HJ, Oh JY. Rapamycin regulates macrophage activation by inhibiting NLRP3 inflammasome-p38 MAPK-NFKB pathways in autophagy- and p62-dependent manners. Oncotarget (2017) 8:40817-31. doi: 10.18632/oncotarget.17256

4. Munk RB, Sugiyama K, Ghosh P, Sasaki CY, Rezanka L, Banerjee $\mathrm{K}$, et al. Antigen-independent IFN- $\gamma$ production by human naïve CD4 T cells activated by IL-12 plus IL-18. PLoS ONE (2011) 6:e18553. doi: 10.1371/journal.pone.0018553

5. Harris J, Hartman M, Roche C, Zeng SG, O'Shea A, Sharp FA, et al. Autophagy controls IL-1beta secretion by targeting pro-IL-1beta for degradation. J Biol Chem. (2011) 286:9587-97. doi: 10.1074/jbc.M110.202911

6. Ziegler-Heitbrock L, Ancuta P, Crowe S, Dalod M, Grau V, Hart DN, et al. Nomenclature of monocytes and dendritic cells in blood. Blood (2010) 116:e74-80. doi: 10.1182/blood-2010-02-258558

\section{Statistical Analysis}

Statistical analysis was performed with Graphpad Prism software (version 6.0; GraphPad, La Jolla, CA). Man-Whitney $T$-test was used for comparison of unpaired values, whereas Wilcoxon paired $T$-test was used for comparison of paired values.

\section{ETHICS STATEMENT}

Consent: This study was carried out in accordance with the recommendations of $\mathrm{CHU}$ Sainte-Justine Institutional Review Board with written informed consent from all subjects. All subjects gave written informed consent in accordance with the Declaration of Helsinki. The protocol was approved by the $\mathrm{CHU}$ Sainte-Justine Institutional Review Board. Written informed consent was obtained from the parents for the publication of this case reports.

\section{AUTHOR CONTRIBUTIONS}

$\mathrm{JB}$ and $\mathrm{ABl}$ took care of the patient, collected data and participated in the manuscript redaction. IF, DD-S, SS, and LM performed experiments and critically read the manuscript. EH took care of the patient, collected data, and critically read the manuscript. ABe designed the study, performed experiments, and critically read the manuscript. FT designed the study, performed experiments, wrote the manuscript, and took care of the patient.

\section{FUNDING}

This work was supported by the starting funds of the CHU Sainte Justine Research Center.

7. Canna SW, Girard C, Malle L, de Jesus A, Romberg N, Kelsen J, et al. Life-threatening NLRC4-associated hyperinflammation successfully treated with IL-18 inhibition. J Allergy Clin Immunol. (2017) 139:1698-701. doi: $10.1016 /$ j.jaci.2016.10.022

8. Rathinam VA, Fitzgerald KA. Inflammasome complexes: emerging mechanisms and effector functions. Cell (2016) 165:792-800. doi: $10.1016 /$ j.cell.2016.03.046

9. Canna SW, de Jesus AA, Gouni S, Brooks SR, Marrero B, Liu Y, et al. An activating NLRC4 inflammasome mutation causes autoinflammation with recurrent macrophage activation syndrome. Nat Genet. (2014) 46:1140-6. doi: 10.1038/ng.3089

10. Zhang L, Chen S, Ruan J, Wu J, Tong AB, Yin Q, et al. Cryo-EM structure of the activated NAIP2-NLRC4 inflammasome reveals nucleated polymerization. Science (2015) 350:404-9. doi: 10.1126/science.aac5789

11. Grom AA, Horne A, De Benedetti F. Macrophage activation syndrome in the era of biologic therapy. Nat Rev Rheumatol. (2016) 12:259-68. doi: $10.1038 /$ nrrheum.2015.179

12. Weiss ES, Girard-Guyonvarc'h C, Holzinger D, de Jesus AA, Tariq Z, Picarsic J, et al. Interleukin-18 diagnostically distinguishes and pathogenically promotes human and murine macrophage activation syndrome. Blood (2018) 131:144255. doi: 10.1182/blood-2017-12-820852

13. Rauch I, Deets KA, Ji DX, von Moltke J, Tenthorey JL, Lee AY, et al. NAIP-NLRC4 inflammasomes coordinate intestinal epithelial cell 
expulsion with eicosanoid and IL-18 release via activation of caspase1 and -8. Immunity (2017) 46:649-59. doi: 10.1016/j.immuni.2017. 03.016

14. Moghaddas F, Zeng P, Zhang Y, Schützle H, Brenner S, Hofmann SR, et al. Autoinflammatory mutation in NLRC4 reveals a leucine-rich repeat (LRR)-LRR oligomerization interface. J Allergy Clin Immunol. (2018) doi: 10.1016/j.jaci.2018. 04.033. [Epub ahead of print].

15. Marsh RA, Haddad E. How i treat primary haemophagocytic $\begin{array}{lllll}\text { lymphohistiocytosis. } & \mathrm{Br} & \mathrm{J} & \text { Haematol. } & \text { (2018) 182:185-99. }\end{array}$ doi: 10.1111/bjh.15274
Conflict of Interest Statement: The authors declare that the research was conducted in the absence of any commercial or financial relationships that could be construed as a potential conflict of interest.

Copyright (C) 2018 Barsalou, Blincoe, Fernandez, Dal-Soglio, Marchitto, Selleri, Haddad, Benyoucef and Touzot. This is an open-access article distributed under the terms of the Creative Commons Attribution License (CC BY). The use, distribution or reproduction in other forums is permitted, provided the original author $(s)$ and the copyright owner(s) are credited and that the original publication in this journal is cited, in accordance with accepted academic practice. No use, distribution or reproduction is permitted which does not comply with these terms. 